\title{
Rydberg Atoms in Uniform Magnetic Fields: Uncovering the Transition from Regularity to Irregularity in a Quantum System
}

\author{
G. Wunner, U. Woelk, I. Zech, G. Zeller, T. Ertl, F. Geyer, W. Schweitzer, and H. Ruder \\ Lehrstuhl für Theoretische Astrophysik, Universität Tübingen, D-7400 Tübingen, Federal Republic of Germany \\ (Received 7 August 1986)
}

\begin{abstract}
We investigate the eigenvalue spectra of hydrogen Rydberg atoms in strong magnetic fields for manifestations of quantum stochasticity and find (i) a smooth transition from a Poisson-type to a Wignertype distribution of level spacings in the range of energy where classical motion becomes increasingly chaotic, (ii) the occurrence of multiple avoided crossings, and (iii) connected with this, an extreme sensitivity of oscillator strengths, and thus of observable spectra, with respect to small variations of an external parameter, viz., the magnetic field strength.
\end{abstract}

PACS numbers: $31.60 .+b, 05.45 .+b, 32.70 . C s$

Studies of the behavior of quantum systems in a range of energy where their classical counterparts undergo transitions from regularity to irregularity, as manifested in phase space by the gradual destruction of invariant tori, to date have largely been restricted to model Hamiltonian systems. Well known examples are the stadium problem, ${ }^{1}$ the quantum Sinai's billiard, ${ }^{2}$ or harmonic oscillators with cubic, ${ }^{3}$ quartic, ${ }^{4}$ or still higher-degree polynomial corrections. ${ }^{5}$ In this Letter we show that phenomena which have turned out characteristic of the onset of "quantum stochasticity" in these model systems can in fact be recovered in the quantal energy spectra of a real physical system, viz., of Rybderg atoms in uniform magnetic fields. This means that one has a simple prototype system at hand in which to study - not only in theory but also in experiment, quantitatively and in detail, and as a function of a continuously tunable external parameter - phenomena that are expected to be typical of the quantum properties of nonintegrable systems in general. The methods of characterization of "chaotic" quantum spectra which we adopt in this Letter are (i) the statistical analysis of fluctuations of energy eigenvalue sequences in the transition region between regularity and irregularity, (ii) the search for multiple avoided crossings of levels in this region, and (iii) the sensitivity of eigenvalues and transition rates to small changes in the perturbation, viz., the magnetic field.

Rydberg atoms in strong microwave fields have been proposed 6 for investigations of "quantum stochasticity," and experimental results for the field ionization of electrically polarized hydrogen Rydberg atoms have recently been compared with classical calculations in the chaotic regime. $^{7}$ We believe that Rydberg atoms in magnetic fields lend themselves even more readily to complete studies of the transition from regularity to irregularity, since quantal calculations are still feasible in the corresponding range of energy. By contrast, quantal calculations of the microwave ionization of Rydberg atoms are prohibitive because huge numbers of bound levels and the continuum must be included.
After separating the trivial azimuthal dependence, the Hamiltonian of an electron under the combined influence of a fixed Coulomb potential and a uniform magnetic field,

$$
H=\mathrm{p}^{2}-2 / r+2 \beta L_{z}+\beta^{2}\left(x^{2}+y^{2}\right)
$$

(in atomic units, $\beta=B / B_{0}$ with $B_{0}=2\left(\alpha m_{e} c\right)^{2} /(e \hbar)$ $\cong 4.70 \times 10^{5} \mathrm{~T}$ ), reduces to that of a nonintegrable system with two degrees of freedom. Computations of the classical trajectories by a number of authors ${ }^{8}$ have established the existence of irregular orbits above a certain energy which depends on the value of the $z$ component of the angular momentum and the strength of the magnetic field. With regard to experiments currently performed with magnetized hydrogen and deuterium Rydberg atoms by the Bielefeld group ${ }^{9}$ we chose their magnetic field strength, $B=6 \mathrm{~T}$, and $L_{z}=0$. To find the range of energy where, in the corresponding classical situation, the transition from quasiperiodicity to chaos occurs, ${ }^{10}$ we first computed classical trajectories and determined the areal fraction of regular orbits in the Poincaré surfaces of section at $z=0$. For $B=6 \mathrm{~T}, L_{z}=0$ we find the transition to occur for energies between $\sim-100$ and -25 $\mathrm{cm}^{-1}$ below the field-free ionization threshold [cf. Fig. $1(\mathrm{a})]$.

By the nonintegrable nature of the Hamiltonian (1), the Schrödinger equation belonging to it can only be solved by numerical means. The numerical approach we take has been described elsewhere. ${ }^{11}$ In the present calculations basis sizes of up to 6400 were handled for determining both eigenvalues and eigenvectors. Accuracies of at least six significant figures were obtained for energy values, and of three significant figures for oscillator strengths. Results for oscillator strengths of $\Delta m=0$ transitions from $2 p_{0}$ to even parity Rydberg states in the $m=0$ subspace as a function of the energy of the final states are shown in Fig. 1(b) in comparison with the corresponding fraction of regular orbits in the Poincaré surface of section at $z=0$. Figure $1(\mathrm{~b})$ conveys a qualitative impression of the increasing complexity of the quan- 

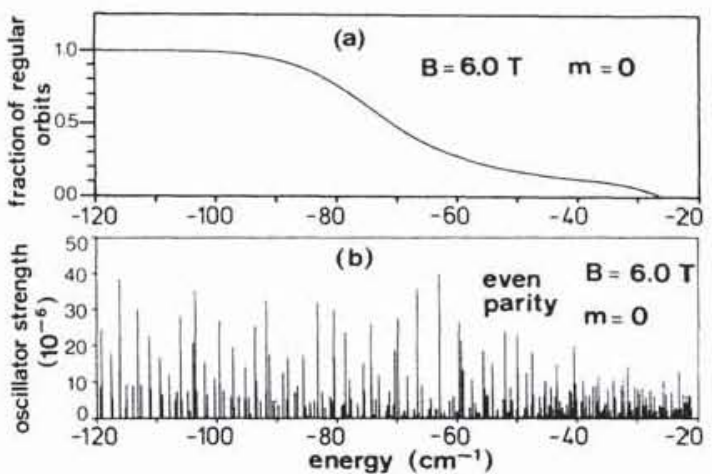

FIG. 1. The fraction of the Poincare surface of section of classical motion at $z=0$ which is filled by regular orbits as a function of orbital energy (a) compared with the oscillator strengths of $\Delta m=0$ transitions from $2 p_{0}$ to hydrogen Rydberg states as a function of the quantal energy of the Rydberg states (b). The breakdown of regularity in classical motion is reflected in an increasing complexity of the quantal spectrum.

tal spectrum as one penetrates into ranges of energy where classical motion becomes more and more chaotic.

To put this observation on a more quantitative footing we undertook a statistical analysis of the level fluctuations of the sequence shown in Fig. 1(b). Following the procedure described by Bohigas, Giannono, and Schmit ${ }^{12}$ we first determined the function $\bar{N}(E)$, which gives the average number of levels up to energy $E$, and replaced every eigenvalue $E_{i}$ by $\varepsilon_{i}=\bar{N}\left(E_{i}\right)$ to obtain a level sequence with constant average spacing, $D$, between adjacent levels. For this sequence nearest-neighbor spacing distributions were calculated. Figure 2 shows histograms of the distributions obtained in three successive energy intervals, along with the corresponding results of the Dyson-Mehta $\Delta_{3}$ statistics. The smooth curves in the histograms represent least-squares fits by the family of functions

$$
P_{2}(S, q)=q^{2} e^{-q S} \operatorname{erfc}\left(\frac{1}{2} \sqrt{\pi} q^{\prime} S\right)+\left(2 q q^{\prime}+\frac{1}{2} \pi q^{\prime 3} S\right) \exp \left(-q S-\frac{1}{4} p q^{\prime 2} S^{2}\right) \quad\left(0 \leq q \leq 1, q^{\prime} \equiv 1-q\right),
$$

which Berry and Robnik ${ }^{13}$ have shown to yield quite generally the correct description of the level spacing distributions in the semiclassical limit for any system with two degrees of freedom when one chaotic region predominates. These functions interpolate between a Poisson distribution (regular case, $q=1$ ) and a Wigner distribution (chaotic case, $q=0$ ). In the problem under consideration the turnover from a Poisson-type to a Wigner-type distribution as one proceeds to energies where classical motion becomes increasingly irregular, is obvious from the curves shown in Fig. 2. In an analogous manner the $\Delta_{3}$ statistics display a transition from the Poisson case to that characteristic of the predictions of random-matrix theory (Gaussian orthogonal ensemble, GOE). The universality of the characterization of the onset of quantum stochasticity by these level fluctuation laws is thus reinforced also in magnetic Rydberg atoms.

We note that our finding is confirmed by very recent, independent work of Wintgen and Friedrich, ${ }^{14}$ and Delande and Gay ${ }^{15}$ for the same system. These authors,
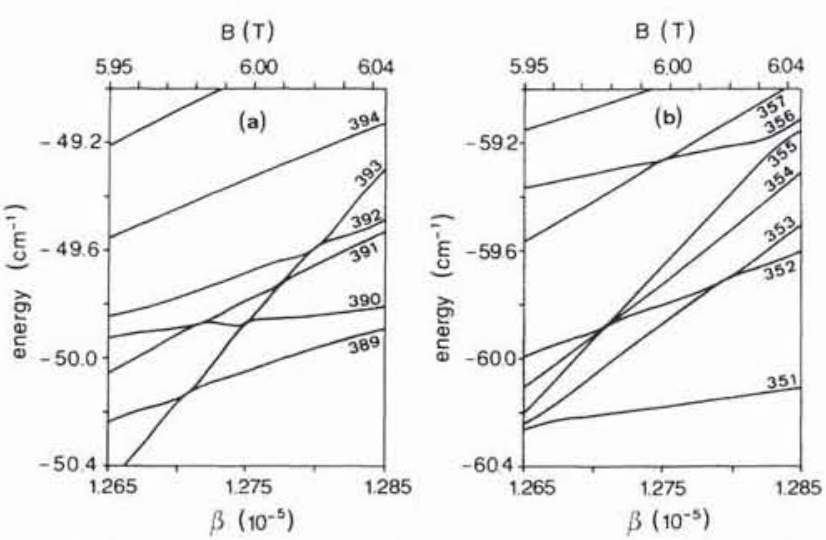

FIG. 3. Energy levels of hydrogen Rydberg states with $m=0$ and even parity as functions of the magnetic field strength in a narrow strip around $6 \mathrm{~T}$. Note the occurrence of rapid successions of (a) avoided crossings and of (b) triple avoided crossings. The numbers attached to the levels indicate the state of excitation of the levels in the $0^{+}$subspace. 


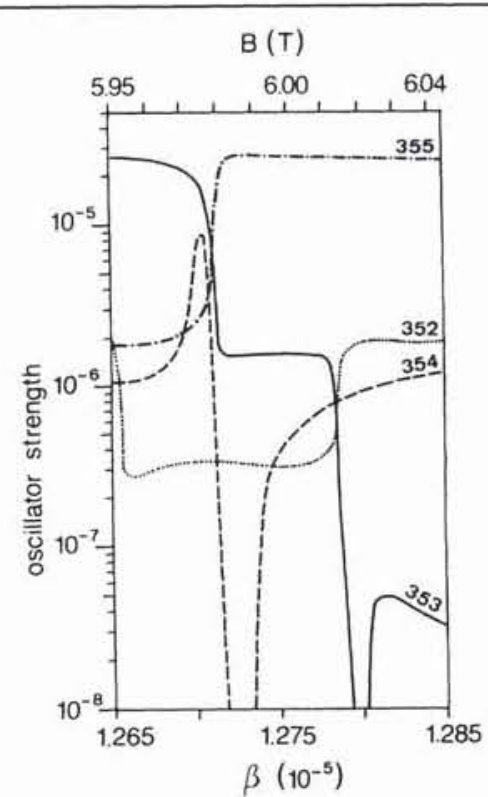

FIG. 4. Oscillator strengths of $\Delta m=0$ Balmer transitions to the levels participating in the triple avoided crossing of Fig 3(b) (numbers $353,354,355$ ) as a function of field strength in a narrow strip around $6 \mathrm{~T}$. The transition strength to level 352 , which goes through an avoided crossing with level 353, is shown for completeness. Note the extreme fluctuations of the line strengths at slight variations of the field.

however, restrict themselves to the limiting cases of complete regularity, or irregularity, while we study the behavior of the system in the transition from the one limit to the other.

A statistical analysis of experimental data for the positions of Rydberg levels in a strong magnetic field is hampered, apart from possible resolution problems, by the fact that transition strengths to levels may become very small, and consequently the missing of these levels in the spectral sequence can considerably counterfeit the level-spacing distribution. Manifestations of quantum stochasticity which lend themselves more unambiguously to direct experimental verification are revealed when we ask about the dependence of positions and intensities of spectral lines belonging to transitions to Rydberg states on the field strength. We have computed, in the transition regime from regularity to irregularity, the energies and wave functions of $m=0$, even-parity states as functions of the field in the interval $5.95 \mathrm{~T} \leq B \leq 6.05 \mathrm{~T}$. Examples for the behavior of the energy levels are shown in Fig. 3 in two ranges of energy. Noteworthy is the occurrence of rapid successions of avoided crossings [Fig. 3(a)], and even of a triple avoided crossing [Fig. 3(b)]. These have been predicted as typical symptoms of quantum stochasticity. ${ }^{16}$ The extreme sensitivity of the actually observable spectra on the field strength is demonstrated by Fig. 4, where we have plotted the oscillator strengths of $\Delta m=0$ Balmer transitions to the levels par- ticipating in the triple avoided crossing of Fig. 3(b) as a function of the magnetic field. This example illustrates that small variations of the field can cause extreme fluctuations of the line strengths and, thus, lead to drastic changes in the outward appearance of the observed spectra. To our knowledge, this is the first time that these manifestations of quantum stochasticity are-theoretically-discovered in this "real" quantum system. The ranges of energy and field are easily accessible in the laboratory, and thus the experimental verification of these symptoms of "quantum chaos" in bound-bound transitions of hydrogen Rybderg atoms in strong magnetic fields is strongly encouraged by our investigation.

This work was supported by the Deutsche Forschungsgemeinschaft. We thank the staff of the Supercomputing Center at the University of Karlsruhe for generously providing computer time on the Cyber 205.

'S. W. McDonald and A. N. Kaufman, Phys. Rev. Lett, 42. 1189 (1979); G. Casati, F. Valz-Gris, and I. Guarneri, Lett. Nuovo Cimento 28, 279 (1980); M. Shapiro and G. Goelmann, Phys. Rev. Lett. 53, 1714 (1984).

${ }^{2}$ O. Bohigas, M. J. Giannono, and C. Schmit, Phys. Rev. Lett. 52, 1 (1984); M. V. Berry, Ann. Phys. (N.Y.) 131, 163 (1981).

${ }^{3}$ D. W. Noid, M. L. Koszykowski, M. Tabor, and R. A. Marcus, J. Chem. Phys. 72, 6169 (1980); D. W. Noid, M. L. Koszykowski, and R. A. Marcus, J. Chem. Phys. 78, 4018 (1983); M. Henon and C. Heiles, Astron. J. 69, 73 (1964).

${ }^{4}$ E. Haller, H. Köppel, and L. S. Cederbaum, Phys. Rev. Lett. 52, 1665 (1984); H. D. Meyer, E. Haller, H. Köppel, and L. S. Cederbaum, J. Phys. A. 17, L831 (1984); Z. Deng and F. T. Hioe, Phys. Rev. Lett. 55, 1539 (1985), 56, 1757(E) (1986).

${ }^{5}$ T. H. Seligman, J. J. M. Verbaarschot, and M. R. Zirnbauer, Phys. Rev. Lett. 53, 215 (1984); T. H. Seligman, J. J. M. Verbaarschot, and M. R. Zirnbauer, J. Phys. A 18, 2751 (1984).

${ }^{6}$ N. B. Delone, B. P. Krainov, and D. L. Shepelyansky, Usp. Fiz. Nauk. 140, 355 (1983) [Sov. Phys. Usp. 26, 551 (1983)]; R. V. Jensen, Phys. Rev. A 30, 386 (1984).

${ }^{7}$ J. E. Bayfield and L. A. Pinnaduwage, Phys. Rev. Lett. 54, 313 (1985); R. V. Jensen, Phys. Rev. Lett. 54, 2057 (1985); K. A. H. van Leeuwen, G. v. Oppen, S. Renwick, J. B. Bowlin, P. M. Koch, R. V. Jensen, O. Rath, D. Richards, and J. G. Leopold, Phys. Rev. Lett. 55, 2231 (1985).

${ }^{8}$ A. R. Edmonds and R. A. Pullen, Imperial College Report No. 28-30, 1979 (to be published); M. Robnik, J. Phys. A 14 3195 (1981); A. Harada and H. Hasegawa, J. Phys. A 16, L259 (1983); J. B. Delos, S. K. Knudson, and D. W. Noid, Phys. Rev. A 30, 1208 (1984).

${ }^{9}$ A. Holle, G. Wiebusch, J. Main, B. Hager, H. Rottke, and K. H. Welge, Phys. Rev. Lett. 56, 2594 (1986); experimental studies of barium Rydberg states in the $n$-mixing regime for fields up to $0.75 \mathrm{~T}$ have recently been reported by $\mathrm{H}$. Rinneberg et al., in Proceedings of the Eighteenth European Group for Atomic Spectroscopy Conference, Marburg, 1986, Europhysics Conference Abstracts 10F, edited by M. Elbel, 
H. Hühnermann, and R. Quad (European Physical Society, Petit-Lancy, Switzerland, 1986), p. 103.

${ }^{10}$ Note that the case $L_{z}=0$ is not contained in the results presented by Delos et al., Ref. 8 .

"W. Rösner, G. Wunner, H. Herold, and H. Ruder, J. Phys. B 17, 29 (1984); G. Wunner, W. Rösner, H. Herold, and H. Ruder, Astron. Astrophys. 149, 102 (1985); G. Wunner, M. Kost, and H. Ruder, Phys. Rev. A 33, 1444 (1985); other numerical approaches to the problem were taken by $\mathrm{C}$. W. Clark and K. T. Taylor, J. Phys. B 13, L737 (1980), 15, 1175 (1982); D. Delande and J. C. Gay, J. Phys. B 16, L335 (1984),
19, L173 (1986); D. Wintgen and H. Friedrich, J. Phys. B 19. 991, L99 (1986).

${ }^{12}$ Bohigas et al., in Ref. 2.

${ }^{13}$ M. V. Berry and M. Robnik, J. Phys. A 17, 2413 (1984).

${ }^{14} \mathrm{D}$. Wintgen and H. Friedrich, Phys. Rev. Lett. 57, 571 (1986).

${ }^{15}$ D. Delande and J. C. Gay, to be published.

${ }^{16} \mathrm{Cf}$. H. S. Taylor, in Atomic Excitation and Recombination in External Fields, edited by M. H. Nayfeh and C. W. Clark (Gordon and Breach, New York, 1985), p. 105, and references therein. 